\title{
Liquefaction Induced Displacement and Rotation of Structures with Wide Basements
}

\author{
F. E. Hughes ${ }^{a, *}$, S. P. G. Madabhushi ${ }^{a}$ \\ ${ }^{a}$ Schofield Centre, University of Cambridge, Cambridge, UK
}

\begin{abstract}
Earthquake induced liquefaction can cause structures with shallow foundations to experience large settlement and rotation, and can cause subsurface structures to uplift. The performance of structures with basements, which intuitively combine these two problems, is not understood. In this paper, data from three dynamic centrifuge tests on structures with wide basements are examined. The ratio of upward to downward vertical forces was varied, and a symmetric and asymmetric superstructure was tested. Digital image correlation was used to capture the soil displacements, providing novel insight into the co-seismic soil-structure interaction. The inclusion of wide basements was shown to reduce the overall settlement of structures by providing an increased uplift force during the liquefied period. For symmetric structures, symmetric soil displacements occurred around the basement during consecutive half-cycles of sinusoidal shaking, resulting in negligible accumulation of rotation. In contrast, significant rotation was accumulated for an asymmetric structure as a result of the $P-\delta$ effect due to the eccentric mass.
\end{abstract}

Keywords:

earthquake, liquefaction, basement, displacement, rotation, centrifuge modelling

\section{Introduction}

There is an increasing tendency for buildings to have basements. Urban centres are becoming densely populated and land prices are rising, making it

\footnotetext{
${ }^{*}$ Corresponding author

Email address: feh22@cam.ac.uk (F. E. Hughes )
} 
desirable to use underground space for parking and storage. The behaviour of structures with basements sited in liquefiable ground is not well understood. Post-earthquake reconnaissance missions following large earthquake events have provided some reports on the performance of structures with basements. After the Niigata 1964 earthquake it was observed that the presence of a basement did not have a significant effect on the settlement ratio, which was defined as the structure settlement divided by the depth of the liquefied layer [1]. Nothing was stated about the rotation of these structures. More recently, differential displacement was observed across structures with basements as a consequence of liquefaction during the 2010-2011 series of earthquakes in Christchurch, New Zealand $[2,3]$. Additional data on the behaviour of structrues with basements is sparse and the co-seismic soilstructure interaction is not understood.

In contrast, the behaviour of structures with shallow foundations resting upon liquefiable layers has been widely investigated in post-earthquake reconnaissance missions $[1,4,2,5]$ and in the laboratory, primarily using dynamic centrifuge modelling $[6,7,8,9]$. Significant structural settlements and rotations have been observed, with the extent of both depending on several parameters including the depth of the liquefiable layer, shaking intensity, foundation bearing pressure and building aspect ratio to name a few. The bearing pressure applied by a structure has been found to cause a bulb of stiffer soil, which does not liquefy, forming beneath the foundation $[10,11,9]$. Decreasing the thickness of liquefied soil beneath the bulb, either by increasing the foundation bearing pressure or decreasing the thickness of the liquefiable layer, has been shown to cause an increase in accelerations transmitted to the structure [9].

Ground improvement methods can be used to reduce liquefaction induced settlement of structures, for example soil improvement [10, 12, 13, 14], reducing the degree of saturation of the soil [15] and improving drainage using vertical drains [16]. However, these methods have the result of increasing the seismic demand of the structure $[15,13,14]$. By reducing the extent of soil liquefaction the natural isolation provided by attenuation of horizontal accelerations in liquefied soil is removed.

Research was carried out to determine whether basement stories can be used to reduce liquefaction induced settlement of structures by providing an uplift force during the liquefied period. Structures with wide basements were investigated. These structures are representative of hotels or shopping malls with a wide ground floor which could have a basement extending below it, 
and a taller central tower above [17].

\section{Centrifuge experiment setup}

This paper examines the results of three dynamic centrifuge experiments that were undertaken using the $10 \mathrm{~m}$ diameter Turner beam centrifuge at the Schofield Centre at the University of Cambridge [18, 19]. The tests were conducted in a rigid container with a Perspex window which allowed digital image correlation to be conducted. Plane strain conditions were replicated. A layer of Duxseal was used at both ends of the container to limit the effect of the rigid boundaries [20]. Absorbing boundaries such as Duxseal layers are only partially effective, particularly when soil loses stiffness during liquefaction events. It was important for this research to have a Perspex sided model container in order to perform digital image correlation. This was a trade off with using a laminar container which would have had the best boundary conditions for this liquefaction problem.

A homogenous layer of loose Hostun HN31 sand was poured by air pluviation using an automatic sand pourer [21]. Properties of the Hostun sand used are as follows: $G_{s}=2.65, d_{50}=0.424 \mathrm{~mm}, e_{\min }=0.555, e_{\max }=1.01$ and $\phi_{\text {crit }}=33^{\circ}[22]$. The target relative density was $44 \%$, equating to a saturated unit weight of $18.8 \mathrm{kNm}^{-3}$. One third of the sand was dyed blue to increase the contrast in the images to improve the precision of the digital image correlation [23]. Arrays of instruments were placed underneath the structure and in the far-field. Piezoelectric accelerometers (Acc), linear variable differential transformers (LVDTs), micro mechanical system accelerometers (MEMS) and pore pressure transducers (PPTs) were used. Equivalent instrument locations were used in all three tests discussed in this paper, and are shown in Figure 1.

The tests were conducted at a centrifugal acceleration of $60 \mathrm{~g}$. The model was saturated with a high viscosity aqueous solution of hydroxypropyl methylcellulose with a target viscosity of $60 \mathrm{cSt}$. A viscous pore fluid was required in order to overcome the inconsistency between the scaling laws of dynamic and seepage time in centrifuge modelling [24]. Saturation was performed using CAM-Sat, an automated, pressure controlled system [25].

Particle image velocimetry analysis was conducted using GeoPIV-RG to obtain displacements of the soil and structure [26, 27]. A MotionBLITZ EoSens mini2 high-speed recording camera, with 2 GByte internal memory, was used to record the cross section visible through the Perspex window. 


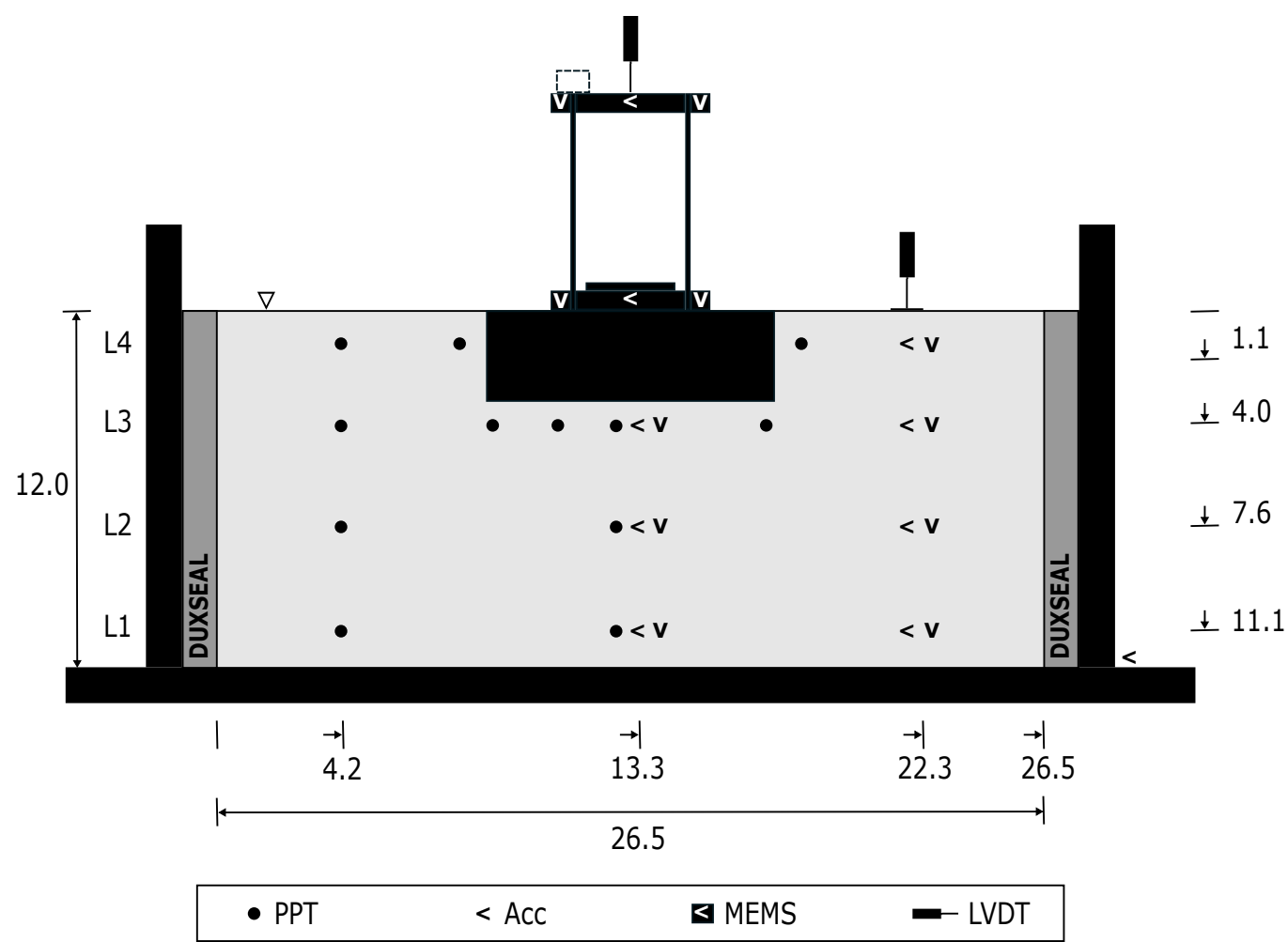

Figure 1: Cross-section sketch of centrifuge experiment layout. Dimensions in metres (prototype scale).

Images were captured at a frame rate of $1068 \mathrm{~Hz}$ for 1.4 seconds at model scale using an exposure time of $120 \mu \mathrm{s}$. The image size was $1696 \times 840$ pixels, corresponding to roughly 4.4 pixels to $1 \mathrm{~mm}$ in model scale. The duration of capturing data at this frame rate was limited by the memory capacity of the camera, therefore images were not captured for the entire post seismic period whilst excess pore pressures were dissipated. However, still images were taken after the dissipation period to observe final displacement and rotation.

\subsection{Ground motion}

A servo-hydraulic earthquake actuator was used to generate one dimensional input motions [28]. The base shaking of the events discussed in this paper were sinusoidal and had the characteristics listed in Table 1. In this table the last row shows the duration of significant shaking, which corre- 


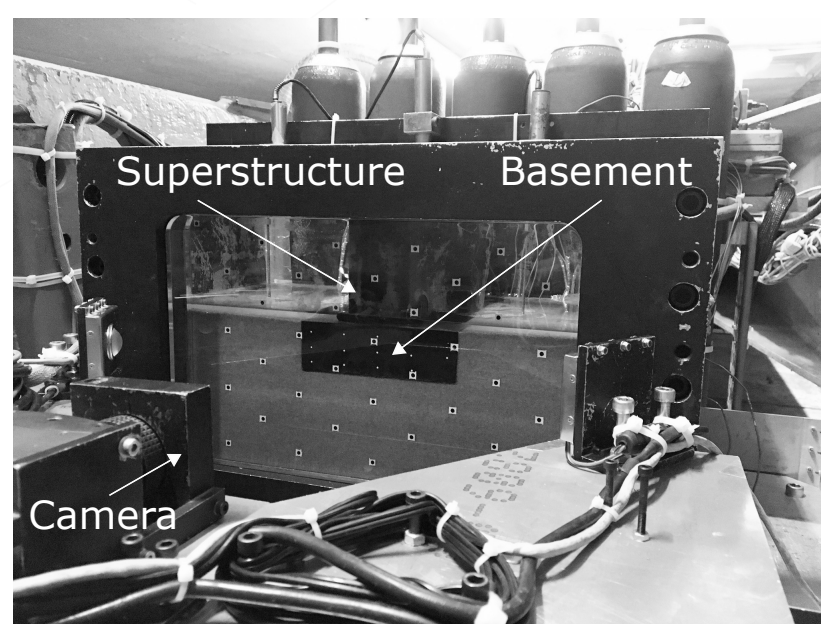

Figure 2: Photograph of centrifuge test setup; Test A loaded onto the Turner beam centrifuge.

sponds to the time between dissipation of $5 \%$ and $95 \%$ of the total Arias intensity [29]. The comparable peak acceleration, number of cycles and Arias intensity indicate that it is possible to directly compare the results of these three tests.

\subsection{Vertical forces}

The basement of the structure was designed to provide an uplift force equal to a specified percentage of the total weight of the structure when the surrounding soil fully liquefied, with the aim to prevent the structure from

Table 1: Characteristics of base shaking (prototype scale).

\begin{tabular}{lllll}
\hline Property & Symbol & Test A & Test B & Test C \\
\hline Peak acceleration $(\mathrm{g})$ & $\mathrm{a}_{\max }$ & 0.392 & 0.424 & 0.359 \\
No of cycles & $\mathrm{N}$ & 20 & 20 & 20 \\
Freq (Hz) & $\mathrm{f}$ & 1 & 1 & 0.88 \\
Input/struct fixed base natural freq & $\mathrm{f} / \mathrm{f}_{n}$ & 1 & 1 & 1 \\
Arias intensity (m/s) & $\mathrm{I}_{a}$ & 9.99 & 11.3 & 10.6 \\
Significant duration (s) & $\mathrm{t}_{5-95}$ & 17.8 & 17.8 & 21.3 \\
\hline
\end{tabular}


settling. This design philosophy is similar to that of floating or compensated foundations used to reduce structure settlement in locations with soft soil conditions [30], and can also be likened to the design of boats.

Vertical forces acting on the structure in static conditions and in the event of complete soil liquefaction are shown in Figure 3. In static conditions, an upward buoyancy force is present $\left(F_{U, H}\right)$ due to the hydrostatic water pressure $\left(u_{h y}\right)$ :

$$
F_{U, H}=u_{h y} \times A=\gamma_{w} d \times A=\gamma_{w} \times V
$$

where $V, A$ and $d$ are the volume, cross sectional area and depth of the basement respectively and $\gamma_{w}$ is the unit weight of water. This uplift force is resisted by the weight of the superstructure and basement $\left(F_{S}\right.$ and $F_{B}$ respectively) and the shear resistance along the soil-structure interface $\left(F_{F}\right)$, as shown in Figure 3. A resultant vertical force acts vertically downwards, which results in the effective bearing pressure given in Table 2.

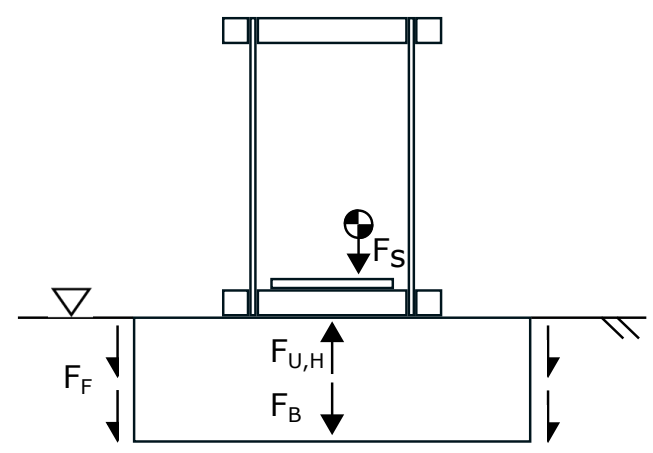

(a)

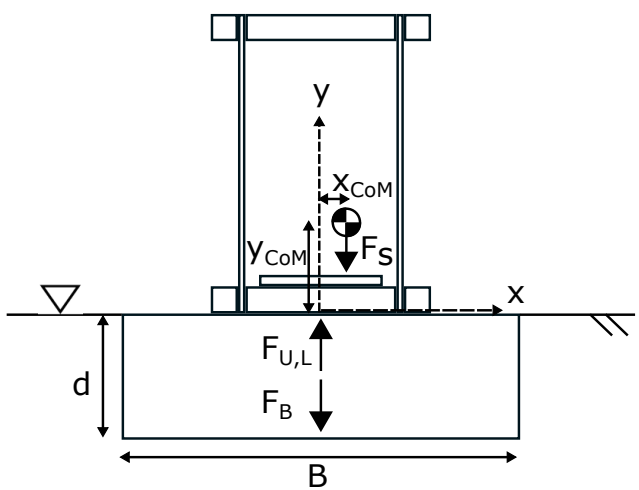

(b)

Figure 3: Vertical forces acting on a structure with a basement (a) static conditions, (b) in the event of complete soil liquefaction. Forces are shown by single-headed arrows. Dimensions are shown by double-headed arrows. References axes are shown by dashed, single-headed arrows.

In the event of complete soil liquefaction, the shear resistance of the soil reduces significantly so the shear resistance along the soil-structure interface is assumed to be negligible [31]. In addition, the uplift force acting on the structure $\left(F_{U, L}\right)$ increases to greater than the hydrostatic value $\left(F_{U, H}\right)$. This can be considered in two ways.

Firstly, excess pore pressures $\left(u_{e x}\right)$ are generated and act on the bottom of the basement in addition to the hydrostatic pressure present in the static 
analysis:

$$
F_{U, L}=F_{U, H}+u_{e x} \times A
$$

When full liquefaction occurs, the excess pore pressure generated becomes equal to the initial vertical effective stress in the soil, and Equation 2 becomes:

$$
F_{U, L}=F_{U, H}+\gamma_{s}^{\prime} d \times A=\left(\gamma_{w}+\gamma_{s}^{\prime}\right) \times V=\gamma_{s} V
$$

where $\gamma_{s}$ and $\gamma_{s}^{\prime}$ are the saturated and buoyant unit weight of the soil respectively.

Alternatively, the total uplift force can be calculated using Archimedes principle, assuming the soil behaves as a dense fluid when liquefied [32]. Archimedes' principle states that any body completely or partially submerged in a fluid at rest is acted upon by an upward, buoyant force, the magnitude of which is equal to the weight of the fluid displaced by the body:

$$
F_{U, L}=\gamma_{s} V
$$

Equations 3 and 4 show that these methods are equivalent when liquefaction occurs and the excess pore pressure generated is equal in magnitude to the initial vertical effective stress in the soil. The ratio of the uplift to total weight when the surrounding soil liquefies (Equation 5) was used as a design parameter for the structures tested in this research:

$$
\frac{U}{W}=\frac{F_{U, L}}{F_{S}+F_{B}}
$$

\subsection{Structural properties}

The model structure consisted of a single degree of freedom (SDOF) sway frame rigidly connected to a rigid basement structure which was constructed out of sheet aluminium surrounding closed-cell foam. Each centrifuge test included one model structure. Structure properties, in prototype scale, are given in Table 2. Total and effective bearing pressures are respectively the total and effective vertical stress applied by the combined superstructure and basement to the soil directly beneath the bottom of the basement. The depth $(2.8 \mathrm{~m})$, breadth $(8.9 \mathrm{~m})$ and length $(11.7 \mathrm{~m})$ of the basement were the same for all three tests. Tests A and B used the same symmetric superstructure but with different total weight of the structure. The structure used in Test $\mathrm{C}$ had the same total weight as that used in Test B, but was asymmetric. A mass was placed on the roof of the structure, centred over one of the 
Table 2: Properties of structures (prototype scale).

\begin{tabular}{lllll}
\hline Property & Symbol & Test A & Test B & Test C \\
\hline Total bearing pressure $(\mathrm{kPa})$ & $\mathrm{q}$ & 68.1 & 55.0 & 55.0 \\
Buoyancy force (static) $(\mathrm{kN})$ & $\mathrm{F}_{U, H}$ & 2860 & 2860 & 2860 \\
Effective bearing pressure (static) $(\mathrm{kPa})$ & $\mathrm{q}^{\prime}$ & 40.1 & 29.0 & 29.2 \\
Ratio of uplift to total weight during liquefaction & $\mathrm{U} / \mathrm{W}$ & 0.75 & 0.93 & 0.92 \\
Symmetric superstructure & & $\mathrm{Y}$ & $\mathrm{Y}$ & $\mathrm{N}$ \\
CoG height above top of basement $(\mathrm{m})$ & $\mathrm{y}_{C o M}$ & -0.044 & 0.526 & 1.37 \\
CoG horizontal distance from centreline $(\mathrm{m})$ & $\mathrm{x}_{C o M}$ & 0 & 0 & -0.184 \\
Fixed base natural frequency $(\mathrm{Hz})$ & $\mathrm{f}_{n}$ & 1 & 1 & 0.83 \\
\hline
\end{tabular}

columns (Figure 1). Symmetry and uniformity are desirable in the design of earthquake resistant buildings, as stated in Eurocode 8 Part 1 (EN19981:2004). However, mass and stiffness eccentricities are inherent in structures due to the presence of features such as water storage tanks, lift shafts and entrances.

All three structures had a ratio of the uplift to total weight when the surrounding soil fully liquefied equal to less than one (Equation 5), implying that, using vertical force equilibrium, all the structures should have a tendency to settle during strong shaking and full liquefaction.

\section{Dynamic behaviour of Basement-Structure system}

\subsection{Excess pore pressures}

The onset of base shaking caused excess pore pressure to be rapidly generated at all instrumented depths beneath each of the structures tested (Figure 4). The excess pore pressure generated increased with increasing depth as the greater initial vertical stress (total and effective) increased the capacity for excess pore pressure generation. The initial vertical effective stress at the instrument locations underneath the centre of each of the structures (shown by the horizontal dashed lines in Figure 4) were calculated by adding the effective bearing pressure applied by the structure to the effective stress caused by the soil between the bottom of the basement and the instrument.

Most previous investigations into earthquake induced liquefaction and soil-structure interaction have used structures with a significantly greater effective bearing pressure than that applied by the structures in these tests, which has prevented full liquefaction occurring directly beneath the structure 

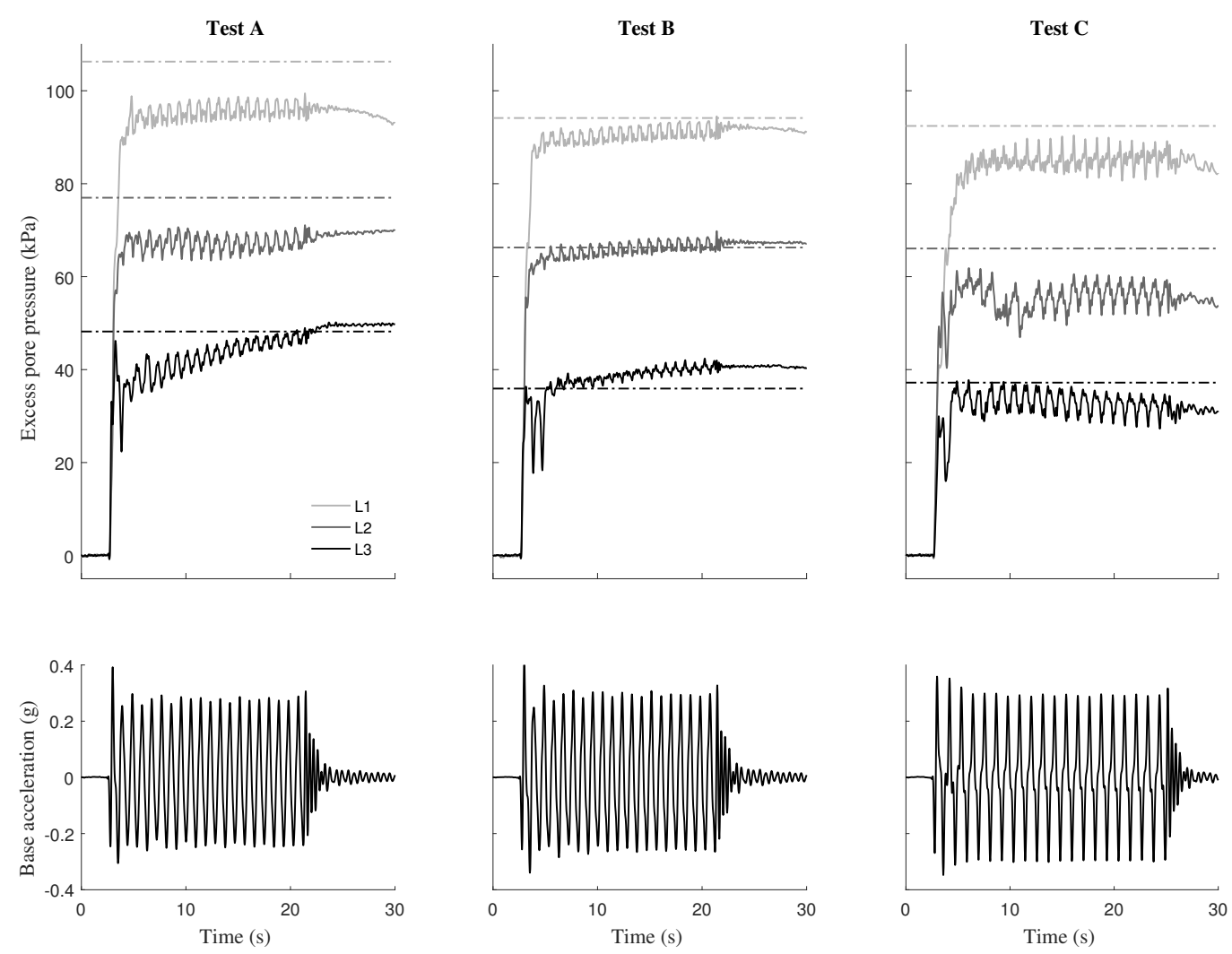

Figure 4: Excess pore pressure generated during the period of base shaking in the instrument array under the centre of the structures. Dashed lines indicate the initial vertical effective stress at the corresponding instrument location.

$[10,7,15,9]$. There are few documented cases for structures with low effective bearing pressure $(<20 \mathrm{kPa})$ resting upon liquefiable soils. The presence of the basement in the tests presented in this paper significantly reduced the effective bearing pressure applied by the structure, to a level below that preventing full liquefaction from occurring. When full liquefaction occurred to the depth of, or deeper than, the bottom of the basement, the effective bearing pressure applied by the structure reduced to $15.9,2.82$ and $3.18 \mathrm{kPa}$ in Tests $\mathrm{A}, \mathrm{B}$ and $\mathrm{C}$ respectively.

\subsection{Accelerations transmitted to the structures}

Generation of excess pore pressure, and the reduction in effective vertical stress that ensues, results in very large stiffness degradation. Transmission of 
horizontally polarised vertically propagating shear waves therefore decrease, causing horizontal accelerations to be attenuated, including in the soil beneath the structure [33]. Horizontal accelerations transmitted to the ground floor of the structure were therefore significantly smaller than those generated by the base shaking (Figure 5). Horizontal accelerations transmitted to the ground floor of the structure were then amplified at the roof level of the SDOF structure, but the amplitude remained smaller than the input base shaking. Accumulated rotation of the structure, which is discussed in detail in Section 3.3, caused the observed drift in the measured horizontal acceleration of the structure in Test C. As the accumulated rotation of the structure increased, the MEMS recorded an increasing component of the centrifugal acceleration.
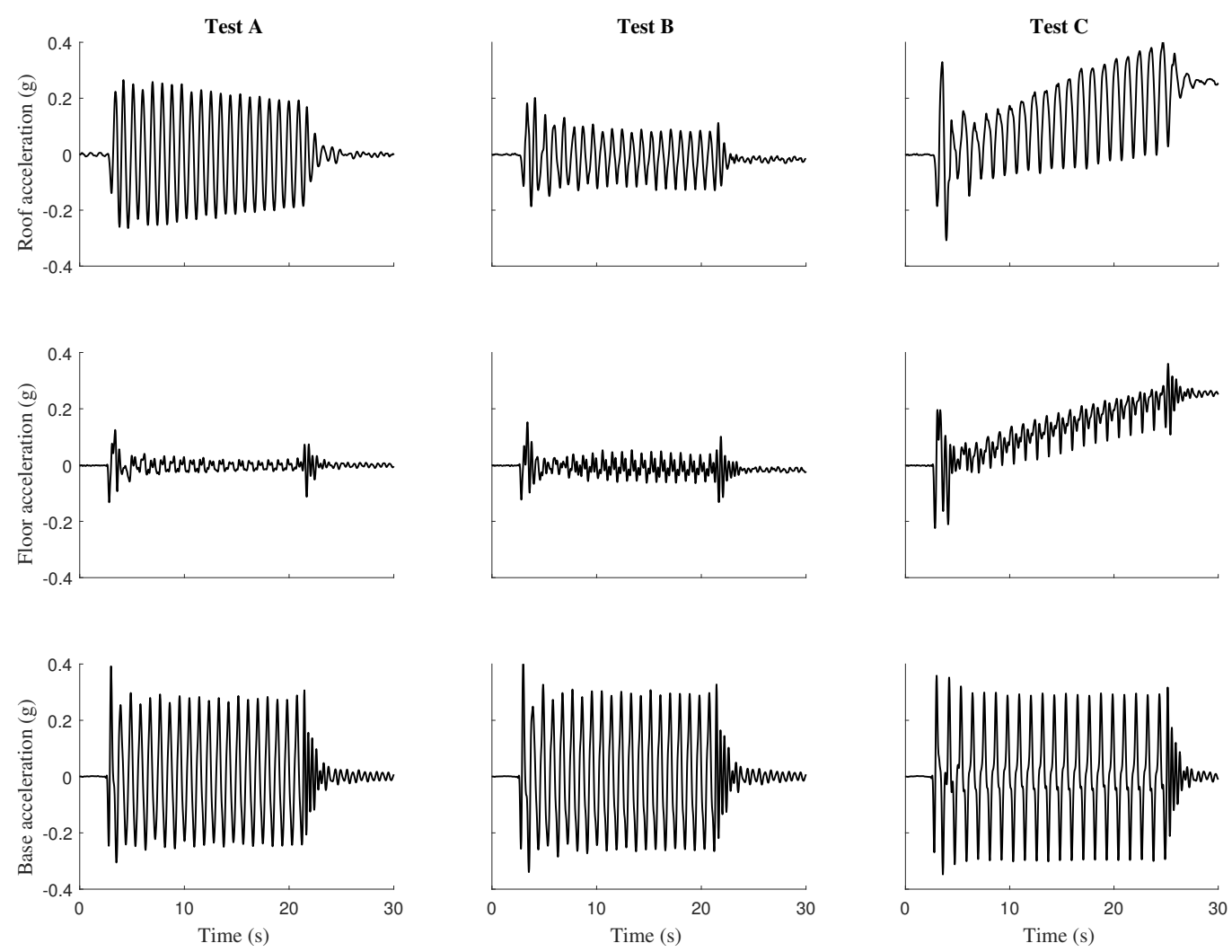

Figure 5: Horizontal accelerations transmitted to the roof (top row) and ground floor (middle row) of the SDOF structure during base shaking (bottom row). 


\subsection{Displacement and rotation of the structure}

The co-seismic vertical and horizontal displacement and rotation of the basement, which was rigidly connected to the ground floor of the superstructure, were obtained using GeoPIV-RG [27] and are shown in Figure 6. Note that the range of the vertical axes is different for the three tests, owing to the very different behaviour observed. The displacements presented are for the centre of the top of the basement/the bottom of the ground floor of the structure. Negative vertical displacement corresponds to settlement of the structure and positive vertical displacement corresponds to uplift of the structure. Anticlockwise rotation is taken to be positive. It is desirable that the structure does not experience displacement or rotation.
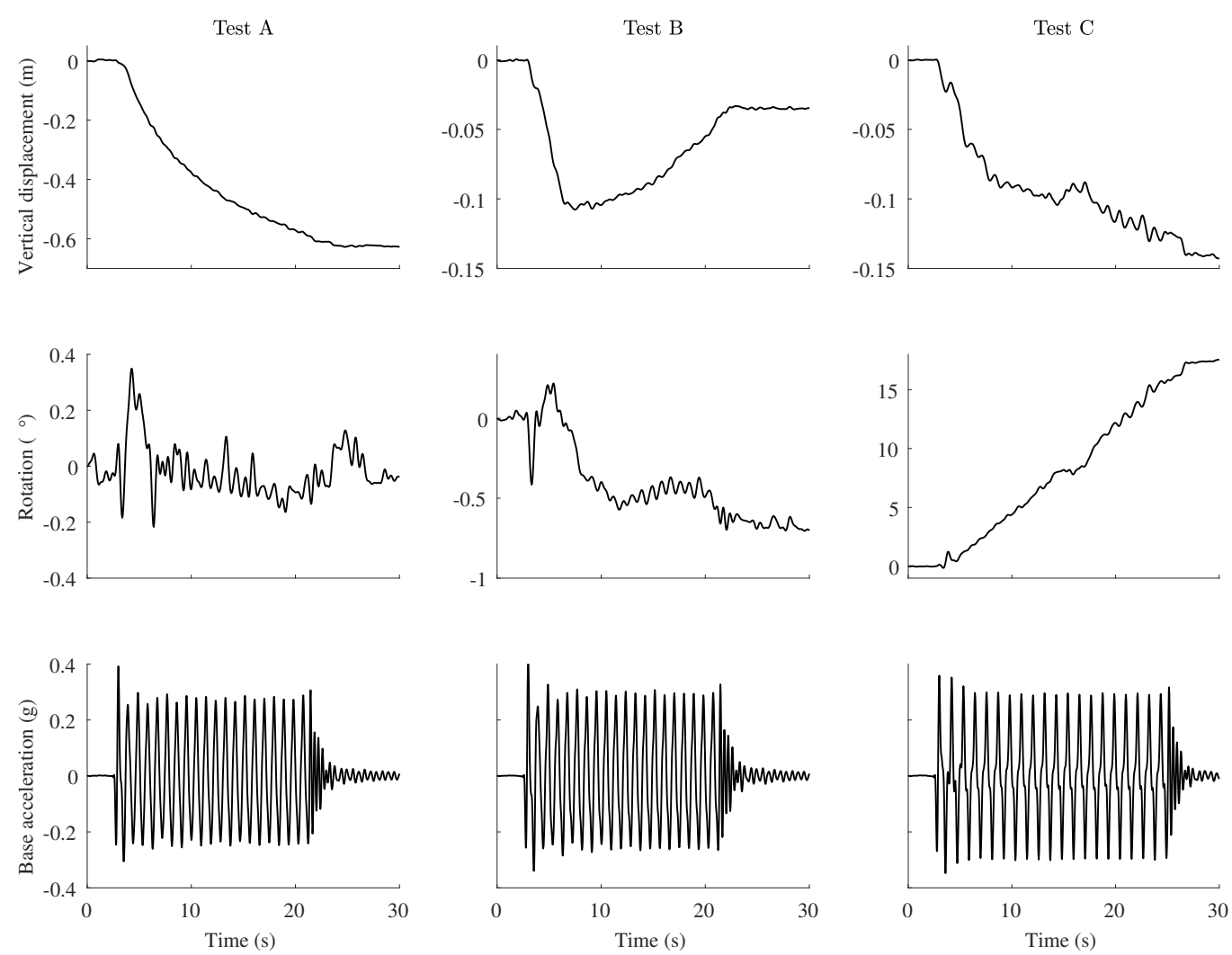

Figure 6: Co-seismic vertical displacement (top row) and rotation (middle row) of the structures tested (prototype scale). Settlement is negative vertical displacement and anticlockwise rotation is positive. Note: the vertical axes do not have the same scale across the three tests. 
The structure in Test A settled during the seismic period, accumulating $632 \mathrm{~mm}$ of vertical displacement by the end of shaking (Figure 6). This is a comparable magnitude to centrifuge testing of structures with shallow foundations, without a basement, sited on deep layers of liquefiable soil $[8,9]$. When shaking occurs, inter storey drift and accumulated rotation of the structure with a basement can cause the vertical forces to no longer be colinear, even for a symmetric structure. The lever arm between the vertical forces results in a couple acting on the structure. Ratcheting, caused by this couple, is anticipated to have had a greater effect on the settlement of the structure with a basement than the reduction in effective bearing pressure due to the buoyancy force provided by the basement.

The structure tested in Test B experienced significantly less vertical displacement. During shaking, the structure initially settled (peak $120 \mathrm{~mm}$ ), before uplifting which reduced the total settlement at the end of shaking to $57 \mathrm{~mm}$. The ratio of the uplift to total weight when the surrounding soil liquefied $(\mathrm{U} / \mathrm{W})$ was greater for the structure in Test $\mathrm{B}$ than Test $\mathrm{A}$, which is likely to have prevented the structure from settling further.

The structure in Test $\mathrm{C}$ accumulated rotation at an approximately constant rate during the shaking, totalling 17.4 degrees at the end of shaking. On average, 0.9 degrees of rotation was accumulated per cycle of sinusoidal shaking. As expected, this rotation occurred in the direction of the eccentric mass. Whilst the centre of the structure did not experience substantial vertical displacement, the sizeable accumulation of rotation resulted in one edge of the structure settling considerably and the other uplifting (Figure 7). In practice this would render the structure unusable and would be particularly problematic for any adjacent structures or utilities.

\section{Soil Deformations}

The region of soil visible through the Perspex window did not include the whole cross section of the model (Figure 7), and did not capture the entire failure mechanism around the basement of each structure. However, the region monitored gave valuable information about the co-seismic soilstructure interaction and how displacement and rotation of the structures developed. The soil displacements shown are relative to the movement of the rigid model container. 

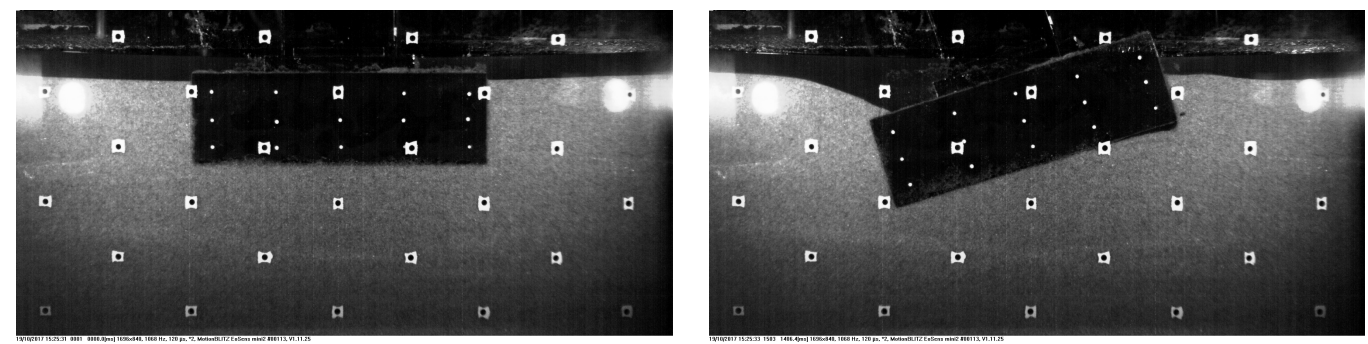

Figure 7: Photos of the cross section of the model visible through the Perspex window, both pre and post Test C.

\subsection{Soil displacements during one cycle of shaking}

Soil displacements accumulated during the 5th and 6th half-cycles of displacement of the model container are shown in Figure 8. The initial cycles are not discussed as the servo-hydraulic earthquake actuator took 2 cycles to settle to a constant amplitude sinusoidal base shaking. The 5th and 6th half-cycles correspond to the time when the settlement rate of Test A was a maximum and the steady accumulation of rotation in Test $\mathrm{C}$ had begun (Figure 6). The model container moved to the right in the 5th half-cycle and then moved to the left in the 6th half-cycle. The 5th and 6th half-cycles of displacement correspond to the 6th and 7th half-cycles of acceleration of the model container respectively.

In Test A, during the 5th half-cycle of displacement the soil underneath the structure moved to the left relative to the rigid base. Soil underneath the basement displaced downwards, with a greater magnitude under the right hand half of the structure. Soil moved upwards to the left of the basement as the structure settled downwards into the soil layer. During the 6th halfcycle, the displacement field was symmetric to that produced in the 5th half-cycle about the vertical line through the centre of the symmetric structure. The symmetric soil displacement in consecutive half-cycles is apparent in the equal amplitude of oscillations in excess pore pressure under the opposite edges of the basement shown in Figure 9. The oscillations were in antiphase, indicating that dilation and contraction spikes were simultaneously occurring under opposite edges of the basement. This implies that the structure was rocking from side to side, with equal amplitude in both directions. Consequently, negligible rotation was accumulated over the two half-cycles. This mechanism is comparable to that generated underneath a structure on shallow foundations sited on a deep layer of liquefiable soil [34], 

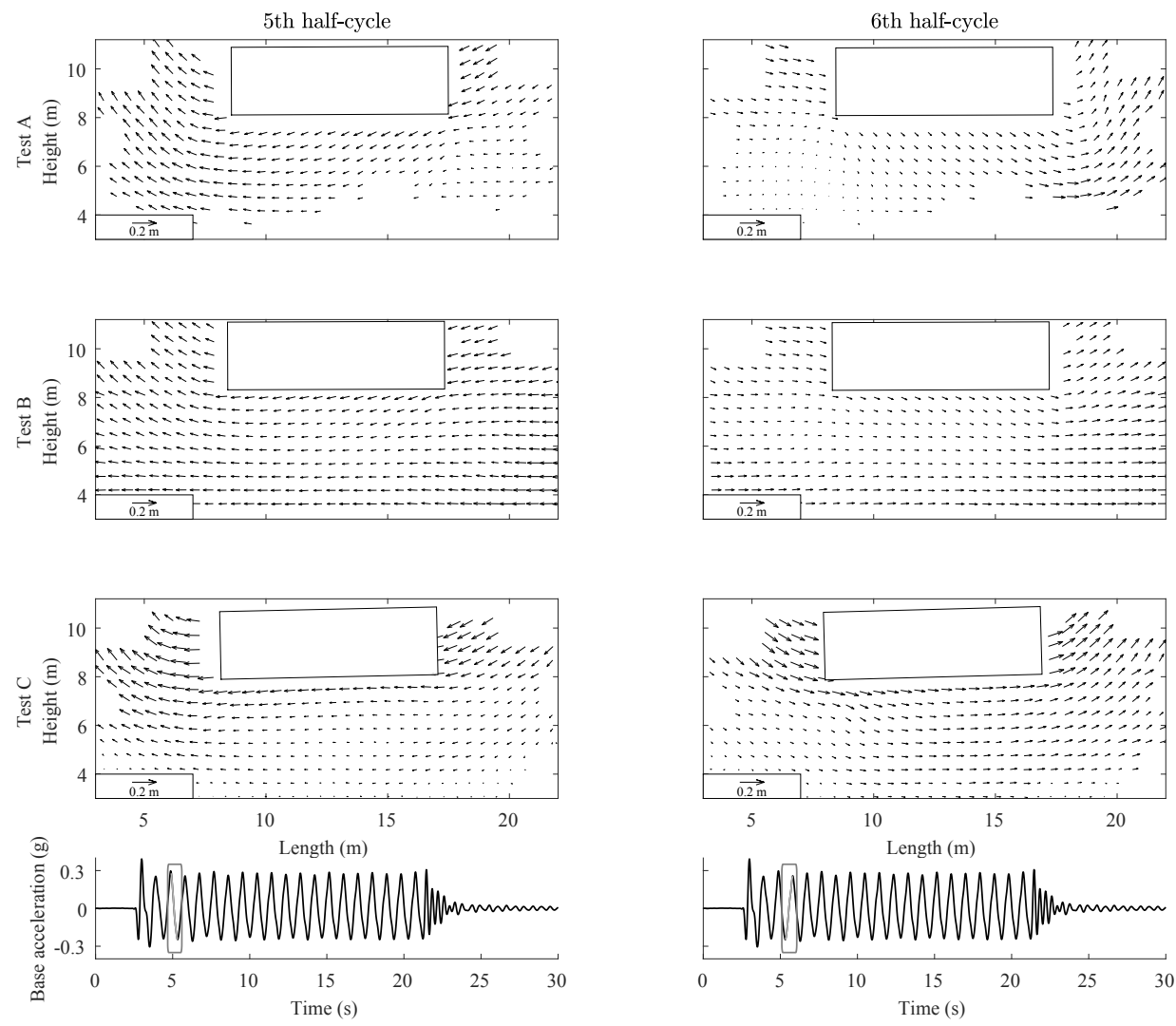

Figure 8: Soil displacement during the 5th (left column) and 6th (right column) half-cycles of displacement of the model container. Location of basement at start of each half-cycle shown. Base shaking for Test A (bottom row), with corresponding half-cycle highlighted in grey. Dimensions in prototype scale. 
and explains why comparable displacements were observed.
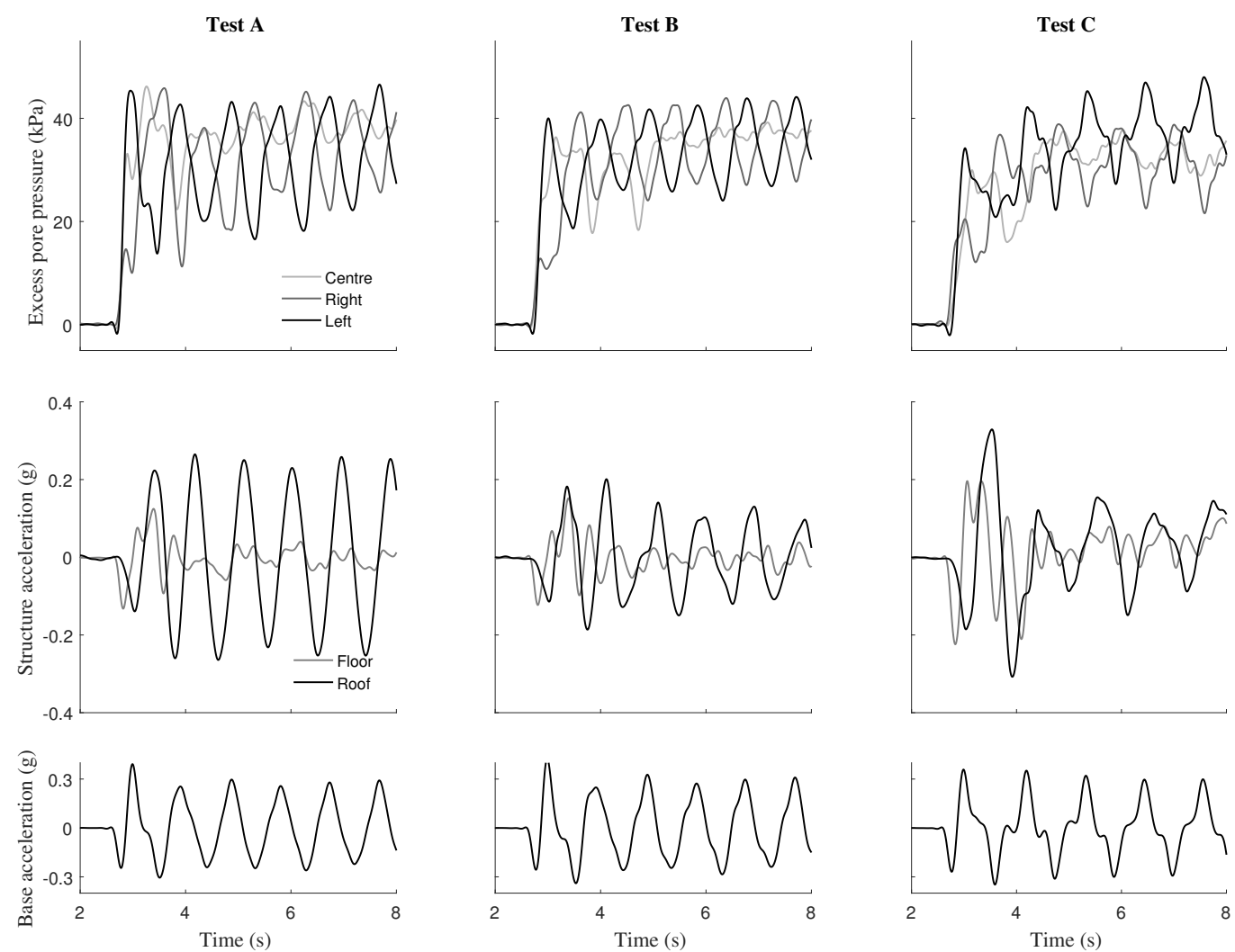

Figure 9: Phase relationships during start of shaking. Top row: excess pore pressures generated below the bottom of the basement. Middle row: absolute acceleration of ground floor and roof of structure. Bottom row: Base shaking.

During Test B, once again the symmetric structure resulted in symmetric displacements of the soil for the two consecutive half-cycles shown. However, the magnitude of displacements was reduced. In contrast to Test $\mathrm{A}$, there was no marked vertical component of downwards soil movement beneath the basement in either half-cycle, resulting in negligible vertical displacement of the structure (Figure 6). Adjacent to the basement, soil movement had a vertical component, which was upwards to the left of the basement for the 5 th half-cycle and to the right for the 6th half-cycle. This was caused by the rocking of the submerged basement and the proximity from the Duxseal at the container boundary may have also contributed to it.

In Test $\mathrm{C}$, the movement of the soil around the basement relative to the 
rigid base was notably larger compared to the previous two tests discussed, shown by the larger displacement vectors in Figure 8. During the 5th halfcycle there was downward movement of soil under the left hand edge of the structure, but no vertical component under the right. During this half-cycle, excess pore pressures measured under the basement show dilation under the left edge and compression under the right (Figure 9). Oscillations are greatest in magnitude under the left, due to the larger soil displacements in this area. In the subsequent half-cycle, there was a considerable downward movement of soil under the left-hand edge again, accompanied by vertically upward movement under the right hand side. Instead of a gap being formed underneath the structure, the liquefied soil moved with the uplifting basement. Sand accumulated below the basement, resulting in accumulation of permanent rotation. The asymmetry in the soil displacements was a result of the geometry of the structure, and the overturning moment generated by the eccentric mass.

\subsection{Total co-seismic soil displacements}

Total co-seismic soil displacements are shown in Figure 10, plotted at the initial location of each tracked patch. In Test A, the soil underneath the centre of the structure moved vertically downwards. Outward displacement of soil increased moving away from the centre of basement towards the edges, and increased with depth. Soil was forced sideways to allow the structure to settle downwards into the soil layer, which caused an upward flow of soil adjacent to the basement. The movement of soil beneath the basement is comparable to that beneath light structures on shallow foundations [9] and the movement of the soil adjacent to the basement has similarities to the flow of liquefied soil around buried pipes [35].

In Test B, soil also flowed upwards adjacent to the basement, but with a significantly smaller magnitude. The soil underneath the basement experience negligible displacement, horizontally or vertically, reflecting the behaviour of the structure (Figure 6).

In contrast, the column of soil beneath the structure in Test $\mathrm{C}$ experienced very large displacements. A semicylinder of soil with a diameter roughly equal to the breadth of the basement rotated with the structure - soil beneath the left hand side displaced downwards and rightwards whilst soil beneath the right hand side displaced upwards. No gap was formed underneath the structure. Negligible displacements were accumulated to the right of the basement, however to the left soil displaced as the structure moved into this 
space. A point of zero displacement formed, likely because of the proximity to the container boundary.
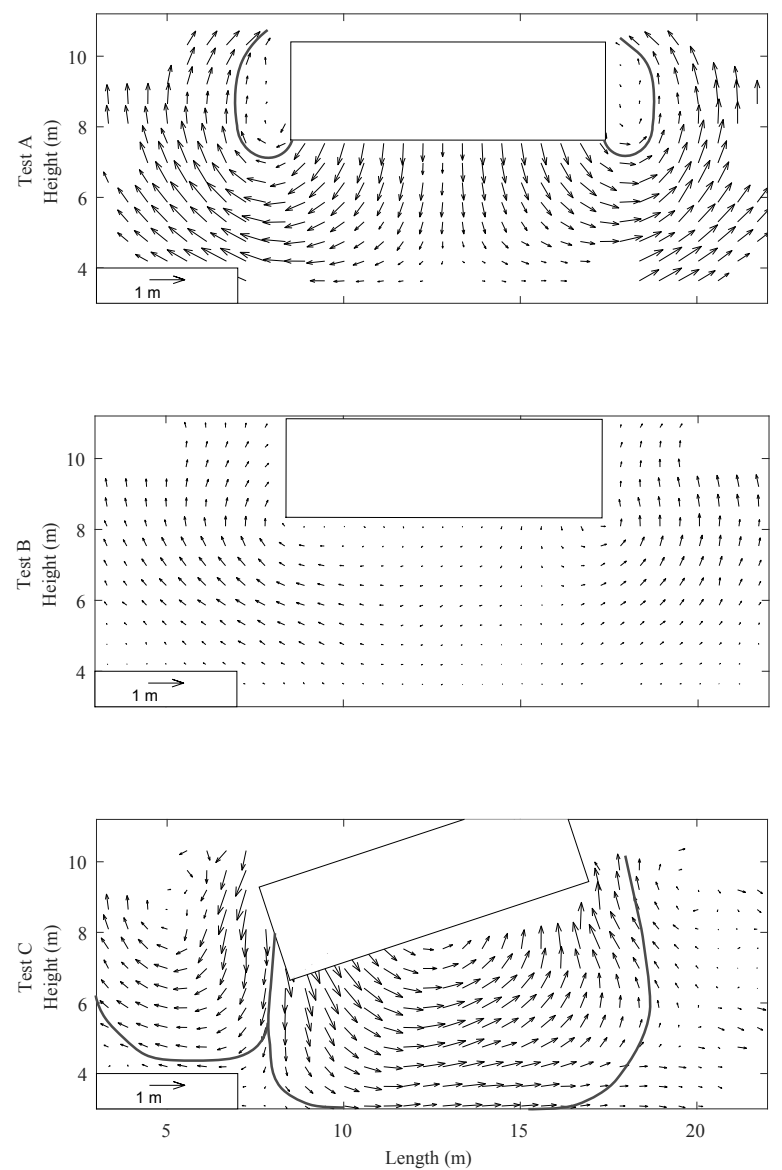

Figure 10: Total co-seismic soil displacement, with failure mechanism drawn in grey for Tests A and C. Location of basement at the end of shaking shown. Dimensions in prototype scale.

\section{Moment transmitted from structure to underlying soil}

The net moment transmitted from the structure to the underlying soil was calculated at each instant a high speed image was recorded $(1068 \mathrm{~Hz}$ at model scale) using Equations 6 to 9. 


$$
M_{\text {net }}(t)=\sum M_{1}(t)+\sum M_{2}(t)-\sum M_{3}(t)
$$

where

$$
\begin{gathered}
M_{1}(t)=\sum m_{i}\left[g+a_{i, y}(t)\right]\left[y_{i} \sin \theta(t)-\left(x_{i}+x_{i, d r i f t}(t)\right) \cos \theta(t)\right]-F_{U}(t)\left[y_{i} \sin \theta(t)\right] \\
M_{2}(t)=\sum m_{i} a_{i, x}(t)\left[y_{i} \cos \theta(t)\right] \\
M_{3}(t)=\sum I_{G i} \ddot{\theta}(t)
\end{gathered}
$$

The structure was split into rigid sections which are shown in Figure 11 - the roof $\left(m_{1}\right)$, the eccentric mass $\left(m_{2}\right)$ (where applicable), and the ground floor and basement $\left(m_{3}\right)$ which were rigidly connected, plus the buoyancy force $\left(F_{U}\right)$. The rigid sections had a mass moment of inertia, $I_{G i}$, about their respective centre of mass located at coordinates $\left(x_{i}, y_{i}\right)$. The net moment applied by the structure consisted of three components. The first and most significant component, $M_{1}(t)$, was the $P-\delta$ effect due to the vertical forces not being co-linear (Equation 7). This was increased by the flexible nature of the structure, where the horizontal displacement of the roof, and when applicable the eccentric mass, lagged behind the ground floor and basement. The second component, $M_{2}(t)$, was provided by the horizontal inertial force of the masses accelerating due to the transmission of the base shaking through the soil body to the structure (Equation 8). The third contribution, $M_{3}(t)$, came from the rotational inertia of the components of the structure (Equation 9 ). The contribution of each rigid section to each component was summed at every time step. Moments were calculated about the centre of the bottom of the basement, point $\mathrm{C}$ in Figure 11. Anticlockwise moments and rotations were taken as positive. Moment-rotation and vertical displacement-rotation graphs are plotted in Figure 12, and are plotted on different horizontal and vertical scales to highlight the different responses of the structures tested.

For the symmetric structures used in Tests A and B, the net co-seismic moments transmitted from the structure to the underlying soil were close to being equal in both the clockwise (negative) and anticlockwise (positive) directions (Figure 12). The amplitude of the moment transmitted was greater for Test A, which had a greater effective bearing pressure. The structures 


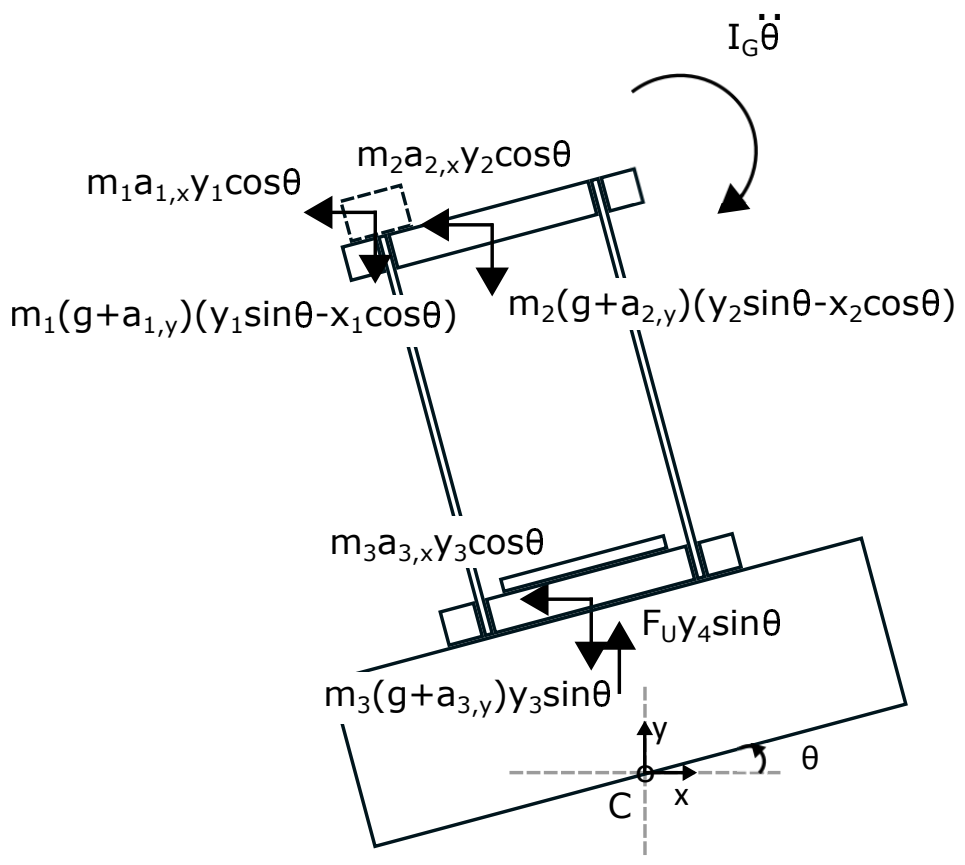

Figure 11: Components of moment transmitted from the structure to the underlying soil. Moments taken about point C. For clarity, shown on a structure with significant accumulated rotation, $\theta$.

were not self righting. However, the sinusoidal shaking, which was of almost equal amplitude in both directions, caused the structures to be re-centred and therefore not accumulate significant rotation during shaking. Negligible rotation was accumulated for Test A, however settlement was accumulated for each cycle of rotation.

The eccentric distribution of weight of the structure in Test $\mathrm{C}$ resulted in a large moment being transferred from the structure to the underlying soil in static conditions. This moment was resisted by the adjacent and underlying soil. During shaking, the peak co-seismic moment transmitted from this structure to the underlying soil occurred during the first cycle of shaking (Figure 12), before the soil was fully liquefied (Figure 4). The peak moment was greatest in the anticlockwise (positive) direction, due to the location of the eccentric mass. Rotation was accumulated in this direction as shaking progressed. Examining one cycle, when the model container moved to the right (5th half-cycle shown in Figure 8), the basement of the structure also moved to the right, however by a smaller magnitude due to the isolation provided 

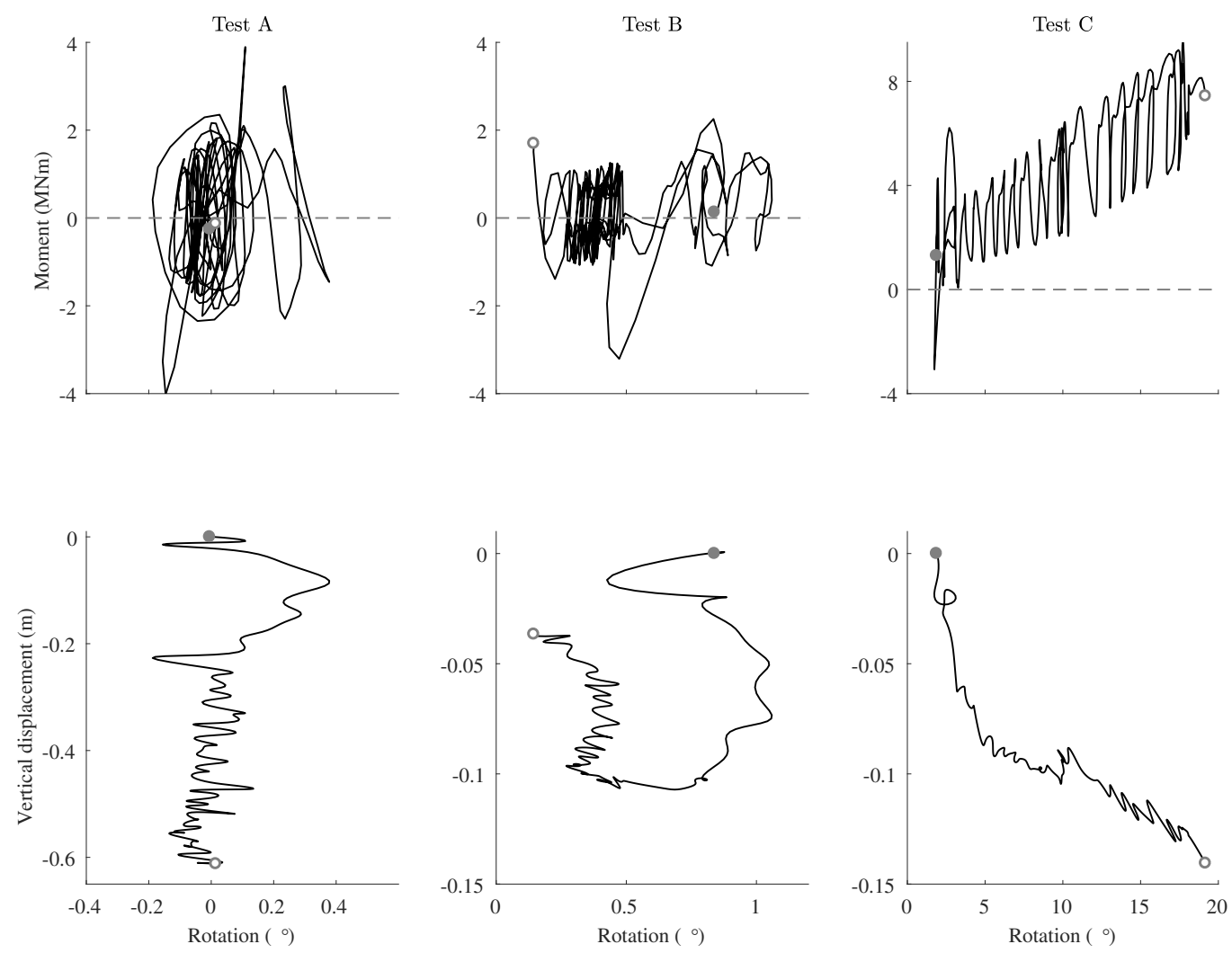

Figure 12: Co-seismic moment - rotation (top row) and vertical displacement - rotation (bottom row) behaviour of the structures (prototype scale). Moments taken about the centre of the bottom of the basement. Anticlockwise moments and rotations are positive. Settlement is negative vertical displacement. Start of base shaking located by filled grey circle and end of base shaking by open grey circle.

by the liquefied soil. The acceleration and displacement of the roof of the structure lagged the input motion. Consequently, the moment caused by the $P-\delta$ effect of the weight of the roof and eccentric mass was at a maximum. As the structure accumulated rotation, this effect became more pronounced and the couple acting on the structure increased. When the model container moved to the left (6th half-cycle shown in Figure 8), the equivalent effect occurred but in the opposite direction. However, the resulting net overturning moment was less than for the previous half-cycle due to the location of the eccentric mass. The structure was not re-centred because of the asymmetry in the moments produced. The greatest contribution of the eccentric mass 
to the moment was through the $M_{1}(t)$ term which increased throughout the earthquake as anticlockwise rotation was accumulated. $M_{2}(t)$ and $M_{3}(t)$ also increased, but not as notably.

The amplitude of rotations presented here for the symmetric structures used in Tests A and B are of the same order of magnitude as those observed for rigid, symmetric structures with shallow foundations on dry sand [36, 37, 38] and on liquefiable layers [39]. However, the moment transmitted from the structure to the underlying soil was far less for structures on shallow foundations than those with basements presented here. The reason for this is twofold. Firstly, and most significantly, the lever arm of all inertia forces was greater since rotation was occurring about a point below the soil surface. The embedment did however increase the moment needed to rotate the structure about its edge due to the passive resistance provided by the soil adjacent to it. Secondly, the moment due to the $P-\delta$ effect was increased since there was an uplift force provided by the basement and also due to interstorey drift of the flexible structure.

Shallow foundations on dry sand have been observed to lift off the soil surface when rocking $[37,38]$. This was not observed in liquefied soil by Adamidis and Madabhushi [39] using shallow foundations or in the tests presented here using structures with basements. As the basement rocked and uplifted, the liquefied soil moved with the uplifting basement. Sand accumulated below the basement, resulting in permanent uplift and rotation of the structure.

\section{Conclusion}

In this paper, the liquefaction induced displacement and rotation of three structures with wide basements has been compared. Digital image correlation enabled the soil displacements to be observed, providing novel insight into the co-seismic soil-structure interaction.

For symmetric structures with wide basements, the uplift force provided by the basement can be used to reduce liquefaction induced settlement. This was achieved for a structure with a ratio of uplift to total weight equal to 0.93 when the surrounding soil fully liquefied. However, when this ratio was reduced to 0.75 settlement was comparable to structures on shallow foundations without basements. Here, increased ratcheting had a greater effect on settlement than buoyancy provided by the basement. In both cases, negligible rotation was accumulated due to symmetric soil displacements occurring 
during consecutive half-cycles of sinusoidal shaking.

For an asymmetric structure with a wide basement, liquefaction resulted in significant rotation and differential displacement. The $P-\delta$ effect due to the eccentric mass resulted in non-symmetric soil displacements beneath the structure. Rotation was accumulated at an approximately constant rate during shaking, resulting in one edge of the structure settling considerably and the other uplifting. A semicylinder of soil with a diameter roughly equal to the breadth of the basement rotated with the structure and experienced large displacements.

The net moment transmitted to the underlying soil by the structures presented here with basements were far greater than those observed for structures without basements. This was primarily due to the increased lever arm of all inertia forces, since rotation was occurring about a point below the soil surface. Additionally, the $P-\delta$ effect was increased since there was an uplift force provided by the basement.

Furthermore, the basements reduced the accelerations transmitted to the structures. The reduction in effective bearing pressure due to the increased uplift force provided by the basement allowed full liquefaction to occur beneath the structure. Horizontal accelerations were therefore attenuated beneath the structure, which naturally isolated the structure from the base shaking and caused a reduction in structure accelerations.

Consequently, based on this research it is possible for basements to be used to reduce liquefaction induced settlement of structures in locations where there is sufficient land available to have a wide basement, and if the mass and stiffness distribution in the structure is reasonably symmetric. Further research is required to establish if narrow basements can also successfully achieve this. Narrow basements would be advantageous in densely populated areas where real estate is at a premium.

\section{Acknowledgements}

The support and assistance provided by the technicians at the Schofield Centre whilst conducting the centrifuge tests was greatly appreciated. The first author would like to thank the Engineering and Physical Science Research Council (EPSRC) for their financial support. 


\section{References}

[1] Y. Yoshimi, K. Tokimatsu, Settlement of buildings on saturated sand during earthquakes, Soils and Foundations 17 (1) (1977) 23-38. doi:10.3208/sandf1972.17.23.

[2] M. Cubrinovski, J. D. Bray, M. Taylor, S. Giorgini, B. Bradley, L. Wotherspoon, J. Zupan, Soil Liquefaction Effects in the Central Business District during the February 2011 Christchurch Earthquake, Seismological Research Letters 82 (6) (2011) 893-904. doi:10.1785/gssrl.82.6.893.

[3] J. D. Bray, R. Luque, Seismic performance of a building affected by moderate liquefaction during the Christchurch earthquake, Soil Dynamics and Earthquake Engineering 102 (August) (2017) 99-111. doi:10.1016/j.soildyn.2017.08.011.

[4] K. Tokimatsu, H. Kojima, S. Kuwayama, A. Abe, S. Midorikawa, Liquefaction-induced damage to buildings in 1990 Luzon earthquake, Journal of Geotechnical Engineering 120 (2) (1994) 290-307. doi:10.1061/(ASCE)0733-9410(1994)120:2(290).

[5] D. Bertalot, A. J. Brennan, F. A. Villalobos, Influence of bearing pressure on liquefaction-induced settlement of shallow foundations, Géotechnique 63 (5) (2013) 391-399. doi:10.1680/geot.11.P.040.

[6] L. Liu, R. Dobry, Seismic Response of Shallow Foundation on Liquefiable Sand, Journal of Geotechnical and Geoenvironmental Engineering 123 (6) (1997) 557-567. doi:10.1061/(ASCE)1090-0241(1997)123:6(557).

[7] S. Dashti, J. D. Bray, J. M. Pestana, M. Riemer, D. Wilson, Mechanisms of Seismically Induced Settlement of Buildings with Shallow Foundations on Liquefiable Soil, Journal of Geotechnical and Geoenvironmental Engineering 136 (1) (2010) 151-164. doi:10.1061/(ASCE)GT.19435606.0000179 .

[8] D. Bertalot, A. J. Brennan, Influence of initial stress distribution on liquefaction-induced settlement of shallow foundations, Géotechnique 65 (5) (2015) 418-428. doi:10.1680/geot.SIP.15.P.002. 
[9] O. Adamidis, S. P. G. Madabhushi, Deformation mechanisms under shallow foundations on liquefiable layers of varying thickness, Géotechnique 68 (7) (2018) 602-613. doi:10.1680/jgeot.17.P.067.

[10] P. A. L. F. Coelho, S. K. Haigh, S. P. G. Madabhushi, T. S. O'Brien, Post-earthquake behaviour of footings employing densification to mitigate liquefaction, Proceedings of the ICE - Ground Improvement 11 (1) (2007) 45-53. doi:10.1680/grim.2007.11.1.45.

[11] B. Ghosh, S. P. G. Madabhushi, Centrifuge modelling of seismic soil structure interaction effects, Nuclear Engineering and Design 237 (8) (2007) 887-896. doi:10.1016/j.nucengdes.2006.09.027.

[12] H. Mitrani, S. P. G. Madabhushi, Cementation liquefaction remediation for existing buildings, Proceedings of the ICE - Ground Improvement 163 (2) (2010) 81-94. doi:10.1680/grim.2010.163.2.81.

[13] J. C. Olarte, S. Dashti, A. B. Liel, B. Paramasivam, Effects of drainage control on densification as a liquefaction mitigation technique, Soil Dynamics and Earthquake Engineering 110 (2018) 212-231. doi:10.1016/j.soildyn.2018.03.018.

[14] J. Olarte, S. Dashti, A. B. Liel, Can ground densification improve seismic performance of the soil-foundation-structure system on liquefiable soils?, Earthquake Engineering and Structural Dynamics 47 (5) (2018) 11931211. doi:10.1002/eqe.3012.

[15] A. Zeybek, S. P. G. Madabhushi, Centrifuge testing to evaluate the liquefaction response of air-injected partially saturated soils beneath shallow foundations, Bulletin of Earthquake Engineering 15 (1) (2016) 339-356. doi:10.1007/s10518-016-9968-6.

[16] B. Paramasivam, S. Dashti, A. Liel, J. Olarte, Effects of Drains on the Performance and Damage Potential of Shallow-Founded Structures, in: 3rd International Conference on Performance-based Design in Earthquake Geotechnical Engineering, Vancouver, Canada, 2017.

[17] A. Y. Elghazouli (Ed.), Seismic design of buildings to Eurocode 8, 2nd Edition, CRC Press, Boca Raton, Florida, 2017. 
[18] A. N. Schofield, Cambridge Geotechnical Centrifuge Operations, Géotechnique 30 (3) (1980) 227-268. doi:10.1680/geot.1980.30.3.227.

[19] A. Schofield, Dynamic and Earthquake Geotechnical Centrifuge Modelling, in: Proceedings of the First International Conference on Recent Advances in Geotechnical Earthquake Engineering and Soil Dynamics, St. Louis, Missouri, US, 1981, pp. 1081-1100.

[20] R. S. Steedman, S. P. G. Madabhushi, Wave propagation in sand medium, in: Proceedings of the 4th International Conference on Seismic Zonation, Stanford, California, 1991.

[21] S. C. Chian, M. E. Stringer, S. P. G. Madabhushi, Use of automatic sand pourers for loose sand models, in: 7th International Conference on Physical Modelling in Geotechnics, Zurich, Switzerland, 2010, pp. $117-121$.

[22] H. Mitrani, Liquefaction Remediation Techniques for Existing Buildings, Ph.D. thesis, University of Cambridge (2006).

[23] S. A. Stanier, D. J. White, Improved image-based deformation measurement in the centrifuge environment, Geotechnical Testing Journal 36 (6) (2013) 915-928. doi:10.1520/GTJ20130044.

[24] S. P. G. Madabhushi, Centrifuge Modelling for Civil Engineers, CRC Press, 2015.

[25] M. E. Stringer, S. P. G. Madabhushi, Improving model quality through computer controlled saturation, in: 7th International Conference on Physical Modelling in Geotechnics, Zurich, Switzerland, 2010, pp. 171176.

[26] D. J. White, W. A. Take, M. D. Bolton, Soil deformation measurement using particle image velocimetry ( PIV ) and photogrammetry, Géotechnique 53 (7) (2003) 619-631. doi:10.1680/geot.2003.53.7.619.

[27] S. A. Stanier, J. Blaber, W. A. Take, D. J. White, Improved imagebased deformation measurement for geotechnical applications, Canadian Geotechnical Journal 53 (5) (2015) 727-739. doi:10.1139/cgj-2015-0253. 
[28] S. P. G. Madabhushi, S. Haigh, N. Houghton, E. Gould, Development of a servo-hydraulic earthquake actuator for the Cambridge Turner beam centrifuge, International Journal of Physical Modelling in Geotechnics 12 (2) (2012) 77-88. doi:10.1680/ijpmg.11.00013.

[29] A. Arias, A measure of earthquake intensity, MIT Press, Cambridge, Massachusetts, 1970.

[30] M. J. Tomlinson, Foundation design and construction, 7th Edition, Prentice Hall, 2001.

[31] J. Koseki, O. Matsuo, Y. Koga, Uplift behaviour of underground structures caused by liquefaction of surrounding soil during earthquake, Soils and Foundations 37 (1) (1997) 97-108. doi:10.3208/sandf.37.97.

[32] F. E. Hughes, S. P. G. Madabhushi, Control of liquefaction induced settlement of buildings using basement structures, in: Proceedings of the 3rd International Conference on Performance-based Design in Earthquake Geotechnical Engineering, Vancouver, Canada, 2017.

[33] F. E. Hughes, S. P. G. Madabhushi, Dynamic Response of a Structure with a Basement Sited on Liquefiable Soil, in: K. Pitilakis (Ed.), 16th European Conference on Earthquake Engineering, Thessaloniki, Greece, 2018.

[34] O. Adamidis, Earthquake-induced liquefaction of sand and response of structures with shallow foundations, Ph.D. thesis, University of Cambridge (2017).

[35] S. C. Chian, K. Tokimatsu, S. P. G. Madabhushi, Soil Liquefaction Induced Uplift of Underground Structures : Physical and Numerical Modeling, Journal of Geotechnical and Geoenvironmental Engineering 140 (10) (2014) 1-18. doi:10.1061/(ASCE)GT.1943-5606.0001159.

[36] S. Gajan, B. L. Kutter, J. D. Phalen, T. C. Hutchinson, G. R. Martin, Centrifuge modeling of load-deformation behavior of rocking shallow foundations, Soil Dynamics and Earthquake Engineering 25 (2005) 773783. doi:10.1016/j.soildyn.2004.11.019. 
[37] S. Gajan, B. L. Kutter, Capacity, Settlement, and Energy Dissipation of Shallow Footings Subjected to Rocking, Journal of Geotechnical and Geoenvironmental Engineering 134 (8) (2008) 1129-1141. doi:10.1061/(ASCE)1090-0241(2008)134:8(1129).

[38] C. M. Heron, S. K. Haigh, S. P. G. Madabhushi, A new macro-element model encapsulating the dynamic momentrotation behaviour of raft foundations, Géotechnique 65 (5) (2015) 442-451. doi:10.1680/geot.SIP.15.P.020.

[39] O. Adamidis, G. S. P. Madabhushi, Rotatonal response of shallow foundations on liquefiable sand, in: K. Pitilakis (Ed.), 16th European Conference on Earthquake Engineering, Thessaloniki, Greece, 2018. 